\title{
Correlation of genotypes for thiopurine methyltransferase and inosine triphosphate pyrophosphatase with long-term clinical outcomes in Korean patients with inflammatory bowel diseases during treatment with thiopurine drugs
}

\author{
Yoon Suk Jung ${ }^{1}$, Jae Hee Cheon ${ }^{1}$, Jae Jun Park ${ }^{1}$, Chang Mo Moon ${ }^{1}$, Eun Soo Kim ${ }^{1}$, Jin Ha Lee \\ Seung Won Kim ${ }^{1}$, Jae Hak Kim², Sung Pil Hong ${ }^{1}$, Tae Il Kim ${ }^{1}$ and Won Ho Kim ${ }^{1}$
}

There is a lack of research describing the associations between thiopurine methyltransferase (TPMT)/inosine triphosphate pyrophosphatase (ITPA) genotypes and long-term clinical outcomes. We investigated whether TPMT/ITPA genotypes predicted long-term clinical response in Korean patients with inflammatory bowel diseases (IBDs) undergoing thiopurine treatment. A total of 204 patients with IBD in whom thiopurine treatment was indicated were enrolled and categorized by TPMT and ITPA genotypes. Long-term follow-up clinical data for these patients were analyzed with specific focus on disease relapse. Of the 204 patients, 162 (79.4\%) patients using thiopurines achieved remission and were included in an analysis of long-term clinical outcomes. There were no significant differences in disease relapse-free survival between wild and mutant types of TPMT $(P=0.903)$ or ITPA $(P=0.392)$, according to the results of the log-rank analysis. Our study suggests that TPMT and ITPA genotypes may not affect the rates of disease relapse in IBD patients treated with thiopurines. Further studies are indicated to confirm the utility of TPMT/ITPA genotyping to guide clinicians formulating individualized treatments for IBD patients requiring thiopurine therapy.

Journal of Human Genetics (2010) 55, 121-123; doi:10.1038/jhg.2009.125; published online 4 December 2009

Keywords: azathioprine; inflammatory bowel disease; inosine triphosphate pyrophosphatase; relapse; thiopurine S-methyl transferase

\section{INTRODUCTION}

Thiopurine drugs, 6-Mercaptopurine (6-MP) and its prodrug azathioprine (AZA), are effective for the induction and maintenance of remission in patients with inflammatory bowel disease (IBD). ${ }^{1,2}$ There has been tremendous interest with regard to thiopurine metabolism as a means of identifying ways in which individual therapy might maximize clinical response and minimize adverse effects. Genetic polymorphisms in the thiopurine $S$-methyl transferase (TPMT) gene have been associated with decreased TPMT activity and the development of myelotoxicity due to high thioguanine metabolite concentrations. ${ }^{3,4}$ Moreover, inosine triphosphate pyrophosphatase (ITPA) deficiency has recently been associated with AZA toxicity because of the accumulation of 6-thioinosine-triphosphate. ${ }^{5,6}$ Theoretically, low TPMT activity might also augment the therapeutic efficacy of thiopurines and lead to better long-term clinical outcomes. To confirm the usefulness of the TPMT/ITPA genotype or TPMT activity in clinical prediction, research regarding the relevance of measurement for prevention of both adverse effects and clinical outcomes is necessary. However, the impact of the TPMT/ITPA genotype on long-term clinical response of IBD patients treated with AZA/6-MP has not yet been examined. In this study, we investigated whether TPMT/ ITPA genotypes could affect long-term clinical outcomes as measured by disease relapse in Korean patients with IBD undergoing AZA or 6-MP treatment.

\section{MATERIALS AND METHODS}

A total of 204 patients with IBD (105 patients with Crohn's disease, 59 with ulcerative colitis and 40 with intestinal Behcet's disease) were initiated on AZA treatment during the study period from June 1999 to November 2008 at Severance Hospital (Seoul, Korea). Patients were initially administered AZA at a fixed dose of $50 \mathrm{mg}$ daily. The dose of AZA might be further increased to $2-3.5 \mathrm{mg} \mathrm{kg}^{-1}$ every 2 weeks if no obvious therapeutic response was observed,

${ }^{1}$ Department of Internal Medicine and Institute of Gastroenterology, Brain Korea 21 Project for Medical Science, Yonsei University College of Medicine, Seoul, Korea and 2Department of Internal Medicine, Dongguk University International Hospital, Goyang, Korea

Correspondence: Dr JH Cheon, Division of Gastroenterology, Department of Internal Medicine, Yonsei University College of Medicine, 134 Shinchon-dong, Seodaemun-gu, Seoul 120-752, Korea. 
provided the patient continued to tolerate therapy and white blood cell counts remained $>3 \times 10^{3}$ per 1 and serum alanine transaminase levels were less than twice the upper limit of normal $\left(50 \mathrm{IU}^{-1}\right)$. If the patient could not tolerate AZA because of adverse effects such as severe gastrointestinal symptoms, 6-MP was substituted for AZA. 6-MP was initially started at $25 \mathrm{mg}$ daily. Similarly, the dose was increased to $1-1.5 \mathrm{mg} \mathrm{kg}^{-1}$ every 2 weeks if no adverse effects such as neutropenia and hepatotoxicity were observed.

Pretreatment and posttreatment clinical data were recorded. The investigators evaluating patients' clinical data were blinded to the results of TPMT and ITPA genotypes. Disease activity was evaluated using the Crohn's disease activity index for Crohn's disease and the partial Mayo score for ulcerative colitis. $^{7,8}$ Patients with intestinal Behcet's disease were considered to have achieved remission or to have relapsed as described previously. ${ }^{9}$

Blood samples for TPMT/ITPA genotyping were collected from patients at the initial clinic visit. Genomic DNA was extracted from blood after informed consent was obtained. Single-nucleotide polymorphisms in the TPMT (exon 5, 7,10 ) and ITPA (exon 2) genes flanking their exon-intron junctions were identified using denaturing high-performance liquid chromatography and subsequent direct sequencing as previously described with some modifications. ${ }^{10,11}$ At the beginning of this study, we conducted a preliminary study by sequencing TPMT/ITPA genes with pilot study samples to discover other polymorphisms, but found that minor allele frequencies were $<5 \%$, which was considered clinically insignificant. Relapse-free survival (time to first relapse) according to TPMT/ITPA genotypes was compared by Kaplan-Meier curves using log-rank analysis. Ethical approval for this study was obtained from the Human Research Review Board of Severance Hospital.

\section{RESULTS}

Table 1 shows the baseline characteristics of the 204 patients enrolled in the study. All patients were initially treated with AZA. However, six patients were unable to tolerate AZA because of adverse effects such as severe gastrointestinal trouble, and were eventually treated with 6-MP. TPMT and ITPA genotypes were assessed in all 204 patients (Table 2). Of the 204 patients studied, 13 were lost to follow-up or discontinued study medications because of side effects. Of the 191 remaining patients who had available medical records regarding clinical response, 164 achieved clinical remission. Of the 164 patients who achieved a remission, 162 received AZA or 6-MP for $\geqslant 3$ months and, were thus included in the final analysis of clinical outcomes in terms of relapse. Starting AZA dose, maximum AZA dose and duration of AZA use were similar between the 'non-relapsers' group and the 'relapsers' group (data not shown). To compare clinical outcomes between wildtype and mutant TPMT and ITPA, Kaplan-Meier survival curves using log-rank analysis were constructed on the basis of the time to first relapse in IBD patients who had received AZA or 6-MP for $\geqslant 3$ months (Figure 1). There were no significant differences in disease relapse-free survival between wild and mutant types of TPMT $(P=0.903)$ or ITPA $(P=0.392)$ according to the results of the logrank analysis. Similarly, on subgroup analysis, there was no significant difference in disease relapse-free survival between wild and mutant types of TPMT or ITPA on each disease entity $(P=0.787$ or $P=0.775$ for Crohn's disease; $P=0.883$ or $P=0.150$ for ulcerative colitis; and $P=0.526$ or $P=0.961$ for intestinal Behcet's disease).

\section{DISCUSSION}

To confirm the usefulness of the TPMT/ITPA genotype in clinical prediction, research regarding the relevance of measurement for prevention of both adverse effects and clinical outcomes is necessary. To our knowledge, this is the first study that is specifically focused on the relationship between TPMT/ITPA genotypes and clinical outcomes during thiopurine treatment.

We found that neither the TPMT nor the ITPA genotype influenced disease relapse in IBD patients treated with thiopurines. In Asian
Table 1 Characteristics of patients enrolled in the study

\begin{tabular}{|c|c|}
\hline Characteristic & No. \\
\hline Total IBD patients & 204 \\
\hline Azathioprine/6-mercaptopurine (\%) & 198/6 (97.1/2.9) \\
\hline $\operatorname{Sex}(M / F)(\%)$ & $114 / 90(55.9 / 44.1)$ \\
\hline Age in years (median, range) & $35(15-71)$ \\
\hline \multicolumn{2}{|l|}{ Type of disease } \\
\hline Crohn's disease (\%) & $105(51.5)$ \\
\hline Ulcerative colitis (\%) & 59 (28.9) \\
\hline Intestinal Behcet's disease (\%) & $40(19.6)$ \\
\hline \multicolumn{2}{|l|}{ Thiopurine indication } \\
\hline Steroid dependent (\%) & 167 (81.9) \\
\hline Steroid refractory (\%) & $2(0.9)$ \\
\hline Fistula (\%) & $35(17.2)$ \\
\hline Starting azathioprine dose in $\mathrm{mg} \mathrm{kg}^{-1}$ (median, range) & $0.93(0.25-3.05)$ \\
\hline Maximum azathioprine dose in $\mathrm{mg} \mathrm{kg}^{-1}$ (median, range) & $1.80(0.53-3.26)$ \\
\hline Treatment length in months (median, range) & $27.0(0.4-150.4)$ \\
\hline Oral 5-aminosalicylic acid use (\%) & $194(95.1)$ \\
\hline
\end{tabular}

Abbreviation: IBD, inflammatory bowel disease.

Table 2 Genotype frequencies of TPMT and ITPA by disease

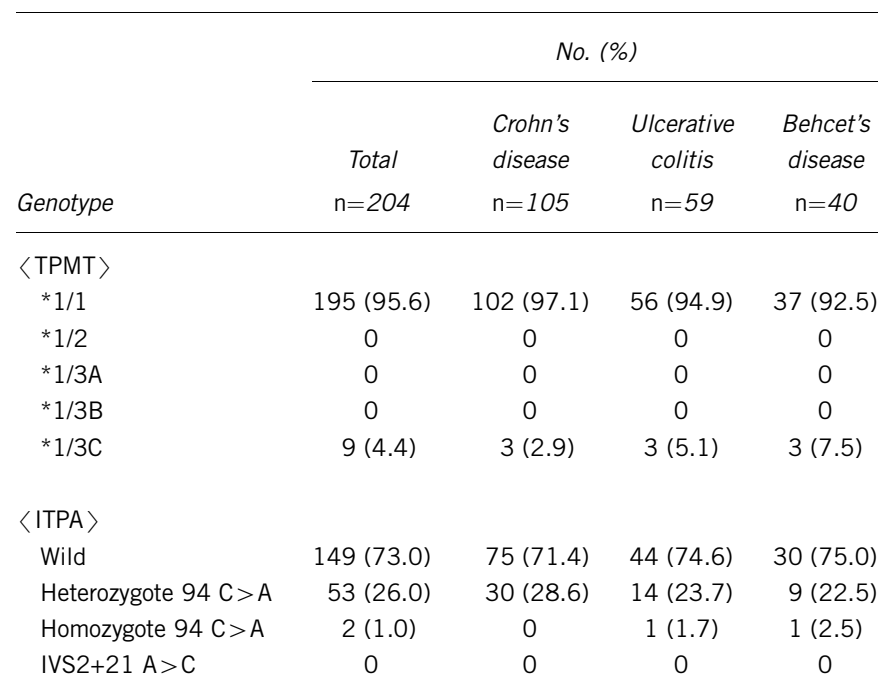

$\overline{\text { Abbreviations: TPMT, thiopurine methyltransferase, ITPA, inosine triphosphate pyrophosphatase. }}$

populations, including Koreans, the frequencies of TPMT mutation are lower than those reported for Western countries, but myelotoxicity develops more frequently. ${ }^{12-14}$ Therefore, determination of the TPMT genotype before the initiation of treatment does not seem to be of significant use for predicting adverse effects with AZA in Asian populations. On the contrary, several studies in Western samples have addressed the impact of TPMT activity and genotype on the clinical response of IBD patients treated with AZA/6-MP and suggested that measurement of TPMT activity might predict clinical response to AZA/6-MP. ${ }^{15,16}$ At present, the reasons for discrepancies among these studies are unclear. However, one possible explanation for these discrepancies is that the Asian population has genetic backgrounds of TPMT that are different from those of the Caucasian 

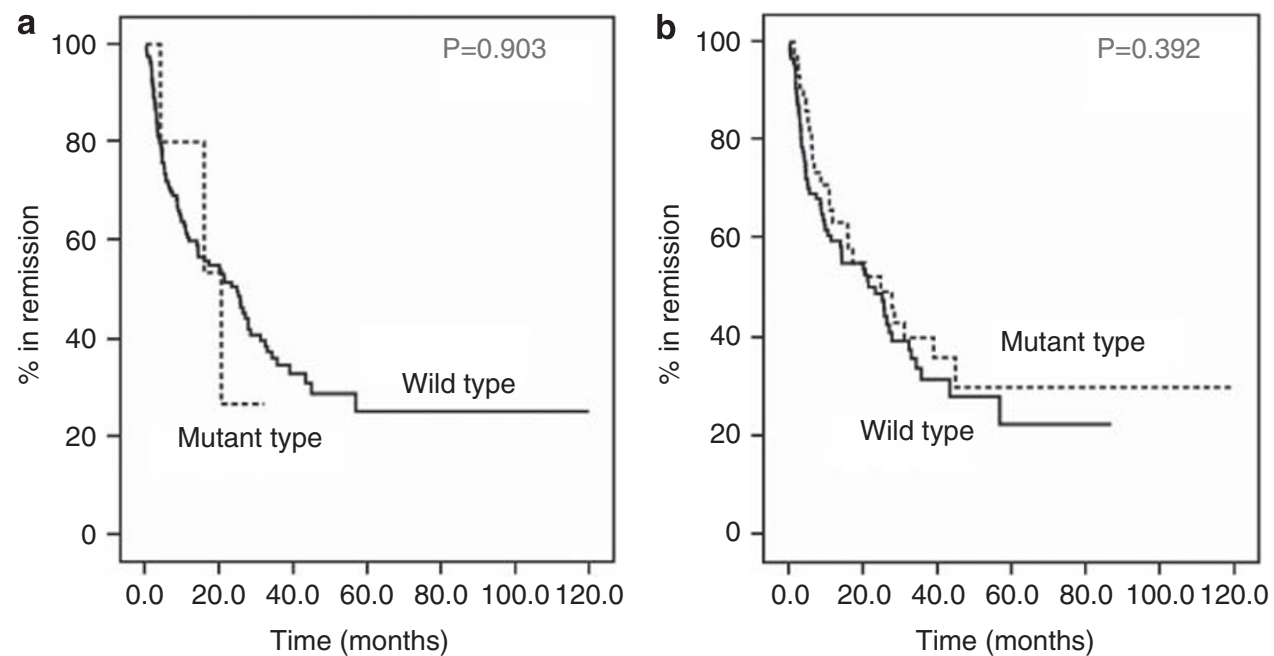

Figure 1 Time to first relapse in IBD patients treated AZA or 6-MP according to the TPMT (a) or the ITPA (b) genotype. IBD, inflammatory bowel disease; AZA, azathioprine; 6-MP, 6-Mercaptopurine; TPMT, thiopurine methyltransferase; ITPA, inosine triphosphate pyrophosphatase.

population. On the basis of the international HapMap data, we compared constructed linkage disequilibrium structures of both TPMT and ITPA genes between Asians and Caucasians, which revealed that definite ethnic diversity was shown in the TPMT gene but not in the ITPA gene (Supplementary Figure 1).

With respect to ITPA, the allele frequency of the ITPA 94C $>$ A mutation in Korean IBD patients was 0.140, higher than that found in Europeans $(0.060-0.070)$ and similar to the frequencies found in Japanese (0.135) and Chinese (0.15) populations. ${ }^{17,18}$ Nevertheless, we did not find any evidence of an association between the ITPA genotype and clinical outcomes. Further research is warranted to reveal the true impact of TPMT/ITPA on the clinical efficacy of thiopurines.

In conclusion, our study suggests that TPMT/ITPA genotypes do not predict relapse in IBD patients using thiopurine treatment. Further large-scale studies should be carried out to validate the usefulness of TPMT/ITPA genotypes for guiding clinicians formulating individualized treatments for patients requiring thiopurine therapy for the treatment of IBD.

\section{CONFLICT OF INTEREST}

The authors declare no conflict of interest.

\section{ACKNOWLEDGEMENTS}

This study was supported by a 2007 Grant (07151kfda676) from the Korea Food and Drug Administration and the Yonsei University College of Medicine, Internal Medicine Research Grant 2009.

1 Present, D. H., Korelitz, B. I., Wisch, N., Glass, J. L., Sachar, D. B. \& Pasternack, B. S Treatment of Crohn's disease with 6-mercaptopurine. A long-term, randomized, doubleblind study. N. Engl. J. Med. 302, 981-987 (1980).

2 Candy, S., Wright, J., Gerber, M., Adams, G., Gerig, M. \& Goodman, R. A controlled double blind study of azathioprine in the management of Crohn's disease. Gut 37, 674-678 (1995).

3 Black, A. J., McLeod, H. L., Capell, H. A., Powrie, R. H., Matowe, L. K., Pritchard, S. C. et al. Thiopurine methyltransferase genotype predicts therapy-limiting severe toxicity from azathioprine. Ann. Intern. Med. 129, 716-718 (1998).
4 Cuffari, C., Dassopoulos, T., Turnbough, L., Thompson, R. E. \& Bayless, T. M. Thiopurine methyltransferase activity influences clinical response to azathioprine in inflammatory bowel disease. Clin. Gastroenterol. Hepatol. 2, 410-417 (2004).

5 Zelinkova, Z., Derijks, L. J., Stokkers, P. C., Vogels, E. W., van Kampen, A. H., Curvers, W. L. et al. Inosine triphosphate pyrophosphatase and thiopurine s-methyltransferase genotypes relationship to azathioprine-induced myelosuppression. Clin. Gastroenterol. Hepatol. 4, 44-49 (2006).

6 Marinaki, A. M., Ansari, A., Duley, J. A., Arenas, M., Sumi, S., Lewis, C. M. et al. Adverse drug reactions to azathioprine therapy are associated with polymorphism in the gene encoding inosine triphosphate pyrophosphatase (ITPase). Pharmacogenetics 14, 181-187 (2004).

7 Sandborn, W. J., Feagan, B. G., Hanauer, S. B., Lochs, H., Löfberg, R., Modigliani, R. et al. A review of activity indices and efficacy endpoints for clinical trials of medical therapy in adults with Crohn's disease. Gastroenterology 122, 512-530 (2002).

8 Lewis, J. D., Chuai, S., Nessel, L., Lichtenstein, G. R., Aberra, F. N. \& Ellenberg, J. H. Use of the noninvasive components of the Mayo score to assess clinical response in ulcerative colitis. Inflamm. Bowel Dis. 14, 1660-1666 (2008).

9 Kim, J. S., Lim, S. H., Choi, I. J., Moon, H., Jung, H. C., Song, I. S. et al. Prediction of the clinical course of Behçet's colitis according to macroscopic classification by colonoscopy. Endoscopy 32, 635-640 (2000).

10 Schaeffeler, E., Lang, T., Zanger, U. M., Eichelbaum, M. \& Schwab, M. Highthroughput genotyping of thiopurine S-methyltransferase by denaturing HPLC. Clin. Chem. 47, 548-555 (2001).

11 Sasaki, T., Goto, E., Konno, Y., Hiratsuka, M. \& Mizugaki, M. Three novel single nucleotide polymorphisms of the human thiopurine S-methyltransferase gene in Japanese individuals. Drug Metab. Pharmacokinet. 21, 332-336 (2006).

12 Jun, J. B., Cho, D. Y., Kang, C. \& Bae, S. C. Thiopurine S-methyltransferase polymorphisms and the relationship between the mutant alleles and the adverse effects in systemic lupus erythematosus patients taking azathioprine. Clin. Exp. Rheumatol. 23, 873-876 (2005).

$13 \mathrm{Kim}$, J. H., Cheon, J. H. \& Kim, W. H. [The frequency and the course of the adverse effects of azathioprine/6-mercaptopurine treatment in patients with inflammatory bowel disease]. Korean J. Gastroenterol. (Taehan Sohwagi Hakhoe chi) 51, 291-297 (2008)

14 Collie-Duguid, E. S., Pritchard, S. C., Powrie, R. H., Sludden, J., Collier, D. A., Li, T. et al. The frequency and distribution of thiopurine methyltransferase alleles in Caucasian and Asian populations. Pharmacogenetics 9, 37-42 (1999).

15 Ansari, A., Hassan, C., Duley, J., Marinaki, A., Shobowale-Bakre, E. M., Seed, P. et al. Thiopurine methyltransferase activity and the use of azathioprine in inflammatory bowel disease. Aliment. Pharmacol. Ther. 16, 1743-1750 (2002).

16 Ansari, A., Arenas, M., Greenfield, S. M., Morris, D., Lindsay, J., Gilshenan, K. et al. Prospective evaluation of the pharmacogenetics of azathioprine in the treatment of inflammatory bowel disease. Aliment. Pharmacol. Ther. 28, 973-983 (2008).

$17 \mathrm{Cao}, \mathrm{H}$. \& Hegele, R. A. DNA polymorphisms in ITPA including basis of inosine triphosphatase deficiency. J. Hum. Genet. 47, 620-622 (2002).

18 Marinaki, A. M., Sumi, S., Arenas, M., Fairbanks, L., Harihara, S., Shimizu, K. et al. Allele frequency of inosine triphosphate pyrophosphatase gene polymorphisms in a Japanese population. Nucleosides Nucleotides Nucleic Acids 23, 1399-1401 (2004).

Supplementary Information accompanies the paper on Journal of Human Genetics website (http://www.nature.com/jhg) 